\title{
Multiple $p$-adic log-gamma functions and their characterization theorem
}

by

\author{
Ken Kamano (Tokyo)
}

1. Introduction and main results. Morita's $p$-adic gamma function $\Gamma_{p}(x)$ is the unique continuous function on the ring of $p$-adic integers $\mathbb{Z}_{p}$ satisfying

$$
\frac{\Gamma_{p}(x+1)}{\Gamma_{p}(x)}= \begin{cases}-x & \left(x \in \mathbb{Z}_{p}^{\times}\right), \\ -1 & \left(x \in p \mathbb{Z}_{p}\right),\end{cases}
$$

and the initial condition $\Gamma_{p}(0)=1$. By 1.1 , we have

$$
\log _{p} \Gamma_{p}(x+1)-\log _{p} \Gamma_{p}(x)= \begin{cases}\log _{p} x & \left(x \in \mathbb{Z}_{p}^{\times}\right), \\ 0 & \left(x \in p \mathbb{Z}_{p}\right),\end{cases}
$$

where $\log _{p}$ is the Iwasawa $p$-adic logarithm. It is easy to see that there is no continuous function $\psi$ on $\mathbb{Z}_{p}$ such that $\psi(x+1)-\psi(x)=\log _{p} x$ for all $x \in \mathbb{Z}_{p} \backslash\{0\}$ (see [10, p. 182]). However, there exists a continuous function $\psi$ on $\mathbb{C}_{p} \backslash \mathbb{Z}_{p}$ satisfying $\psi(x+1)-\psi(x)=\log _{p} x$ for all $x \in \mathbb{C}_{p} \backslash \mathbb{Z}_{p}$ where $\mathbb{C}_{p}$ is the completion of the algebraic closure of the $p$-adic field $\mathbb{Q}_{p}$. An example of such a function is Diamond's $p$-adic log-gamma function $\log \Gamma_{\mathrm{D}}(x)$, which is defined by

$$
\log \Gamma_{\mathrm{D}}(x)=\int_{\mathbb{Z}_{p}}\left((x+t) \log _{p}(x+t)-(x+t)\right) d t \quad\left(x \in \mathbb{C}_{p} \backslash \mathbb{Z}_{p}\right),
$$

where $\int_{\mathbb{Z}_{p}} f(t) d t$ is the Volkenborn integral of $f$ defined by

$$
\int_{\mathbb{Z}_{p}} f(t) d t=\lim _{N \rightarrow \infty} p^{-N} \sum_{a=0}^{p^{N}-1} f(a)
$$

(see Section 2). This function satisfies the expected difference equation

$$
\log \Gamma_{\mathrm{D}}(x+1)-\log \Gamma_{\mathrm{D}}(x)=\log _{p} x
$$

2010 Mathematics Subject Classification: Primary 11S80; Secondary 11B68.

Key words and phrases: p-adic log-gamma function, Volkenborn integral, multiple Bernoulli polynomials. 
for all $x \in \mathbb{C}_{p} \backslash \mathbb{Z}_{p}$. Although the difference equation $(1.2)$ cannot characterize the function $\log \Gamma_{\mathrm{D}}(x)$, Cohen and Friedman [3] proved that 1.2 and a certain integro-differential equation characterize it.

Theorem 1.1 (Cohen-Friedman [3, Section 1]). Diamond's p-adic loggamma function $\log \Gamma_{\mathrm{D}}(x)$ satisfies

$$
\int_{\mathbb{Z}_{p}} \log \Gamma_{\mathrm{D}}(x+t) d t=(x-1)\left(\log \Gamma_{\mathrm{D}}\right)^{\prime}(x)-x+\frac{1}{2} \quad\left(x \in \mathbb{C}_{p} \backslash \mathbb{Z}_{p}\right) .
$$

It is the unique strictly differentiable function $f: \mathbb{C}_{p} \backslash \mathbb{Z}_{p} \rightarrow \mathbb{C}_{p}$ satisfying the difference equation

$$
f(x+1)-f(x)=\log _{p} x
$$

and the Volkenborn integro-differential equation

$$
\int_{\mathbb{Z}_{p}} f(x+t) d t=(x-1) f^{\prime}(x)-x+\frac{1}{2} .
$$

As stated in [3, Section 1], formula (1.3) above can be regarded as a $p$-adic analogue of Raabe's classical formula:

$$
\int_{0}^{1} \log \left(\frac{\Gamma(x+t)}{\sqrt{2 \pi}}\right) d t=x \log x-x \quad(x>0),
$$

where $\log x$ is the ordinary logarithm function on $\mathbb{R}$.

There are many studies on multiple analogues of the gamma function and the log-gamma function. In the complex case, around 1900, Barnes studied multiple gamma functions which are defined by using multiple Hurwitz zeta functions (see e.g. [1]). Vignéras [12] redefined these multiple gamma functions to be functions satisfying a Bohr-Mollerup type theorem. These functions have many applications. For example, Shintani [11] used the double gamma function to study Kronecker's limit formula for certain algebraic fields. In the $p$-adic case, Cassou-Noguès [2] defined multiple $p$-adic log-gamma functions. Variants of multiple $p$-adic log-gamma functions have also been investigated by many authors (e.g. Imai [6] and Kashio [7]).

In this paper, we focus on a simple multiple analogue of Diamond's $p$ adic log-gamma function, denoted by $\log \Gamma_{\mathrm{D}, r}(x)$. For more general forms of these functions $\log \Gamma_{\mathrm{D}, r}(x)$, see Cassou-Noguès [2, p. 53] and Kashio [7, Section 5]. The function $\log \Gamma_{\mathrm{D}, r}(x)(r \geq 1)$ satisfies the difference equation $\log \Gamma_{\mathrm{D}, r}(x+1)-\log \Gamma_{\mathrm{D}, r}(x)=\log \Gamma_{\mathrm{D}, r-1}(x)$ for all $x \in \mathbb{C}_{p} \backslash \mathbb{Z}_{p}$ (Proposition 3.4). As a main result, we show that the function $\log \Gamma_{\mathrm{D}, r}(x)$ satisfies a Raabe-type formula and a characterization theorem. This result is a generalization of Theorem 1.1 because $\log \Gamma_{\mathrm{D}, 1}(x)=\log \Gamma_{\mathrm{D}}(x)$ and $\log \Gamma_{\mathrm{D}, 0}(x)=\log _{p} x$. 
MAIN TheOREM.

(i) For a positive integer $r$ and $x \in \mathbb{C}_{p} \backslash \mathbb{Z}_{p}$, we have

$$
r \int_{\mathbb{Z}_{p}} \log \Gamma_{\mathrm{D}, r}(x+t) d t=(x-r)\left(\log \Gamma_{\mathrm{D}, r}\right)^{\prime}(x)-S_{r}(x),
$$

where $S_{r}(x) \in \mathbb{Q}[x]$ is the multiple Bernoulli polynomial defined by (2.8.

(ii) For a positive integer $r$, the multiple $p$-adic log-gamma function $\log \Gamma_{\mathrm{D}, r}(x)$ is the unique strictly differentiable function $f: \mathbb{C}_{p} \backslash \mathbb{Z}_{p} \rightarrow$ $\mathbb{C}_{p}$ satisfying the following conditions:
(A) $f(x+1)-f(x)=\log \Gamma_{\mathrm{D}, r-1}(x)$.
(B) $r \int_{\mathbb{Z}_{p}} f(x+t) d t=(x-r) f^{\prime}(x)-S_{r}(x)$.

The plan of this paper is as follows. In Section 2, we review Volkenborn integrals and multiple Bernoulli polynomials. In Section 3, we define multiple $p$-adic log-gamma functions $\log \Gamma_{\mathrm{D}, r}(x)$ on $\mathbb{C}_{p} \backslash \mathbb{Z}_{p}$ and give some properties of them. In Section 4, we prove our Main Theorem. In the last Section 5 , we deal with multiple $p$-adic log-gamma functions on $\mathbb{Z}_{p}$. They are generalizations of the logarithm of Morita's $p$-adic gamma function.

2. Multiple Bernoulli polynomials. For a positive integer $n$, we set

$$
\nabla^{n} \mathbb{Z}_{p}=\left\{\left(x_{1}, \ldots, x_{n}\right) \in \mathbb{Z}_{p}^{n} \mid x_{i} \neq x_{j} \text { if } i \neq j\right\} .
$$

The $n$th (order) difference quotient $\Phi_{n} f: \nabla^{n+1} \mathbb{Z}_{p} \rightarrow \mathbb{C}_{p}$ of a function $f$ : $\mathbb{Z}_{p} \rightarrow \mathbb{C}_{p}$ is inductively given by $\Phi_{0} f=f$ and for $\left(x_{1}, \ldots, x_{n+1}\right) \in \nabla^{n+1} \mathbb{Z}_{p}$ by

$$
\Phi_{n} f\left(x_{1}, \ldots, x_{n+1}\right)=\frac{\Phi_{n-1} f\left(x_{1}, x_{3}, \ldots, x_{n+1}\right)-\Phi_{n-1} f\left(x_{2}, x_{3}, \ldots, x_{n+1}\right)}{x_{1}-x_{2}} .
$$

A function $f$ is called a $C^{n}$-function if $\Phi_{n} f$ can be extended to a continuous function $\bar{\Phi}_{n} f: \mathbb{Z}_{p}^{n+1} \rightarrow \mathbb{C}_{p}$. The set of all $C^{n}$-functions from $\mathbb{Z}_{p}$ to $\mathbb{C}_{p}$ is denoted by $C^{n}\left(\mathbb{Z}_{p} \rightarrow \mathbb{C}_{p}\right)$. Moreover, we set $C^{\infty}\left(\mathbb{Z}_{p} \rightarrow \mathbb{C}_{p}\right)=\bigcap_{n=1}^{\infty} C^{n}\left(\mathbb{Z}_{p} \rightarrow \mathbb{C}_{p}\right)$ (e.g. [10, Section 29]). We note that $C^{1}$-functions and strictly differentiable functions are exactly the same.

For a function $f \in C^{1}\left(\mathbb{Z}_{p} \rightarrow \mathbb{C}_{p}\right)$, the limit value

$$
\lim _{N \rightarrow \infty} p^{-N} \sum_{a=0}^{p^{N}-1} f(a)
$$

exists. It is called the Volkenborn integral of $f$ and is denoted by

$$
\int_{\mathbb{Z}_{p}} f(t) d t
$$


(e.g. [10, p. 167]). For a continuous function $f: \mathbb{Z}_{p} \rightarrow \mathbb{C}_{p}$, we denote the indefinite sum of $f$ by $S f$, that is, $S f$ is the unique continuous function on $\mathbb{Z}_{p}$ satisfying $S f(n)=\sum_{j=0}^{n-1} f(j)$ for any positive integer $n$ (e.g. [10, p. 106]). For $f \in C^{1}\left(\mathbb{Z}_{p} \rightarrow \mathbb{C}_{p}\right)$ and $x \in \mathbb{Z}_{p}$, the following identities are known (cf. [10, p. 168]):

$$
\begin{aligned}
& \int_{\mathbb{Z}_{p}} f(x+t) d t=(S f)^{\prime}(x), \\
& \int_{\mathbb{Z}_{p}} f(x+t) d t-\int_{\mathbb{Z}_{p}} f(t) d t=\left(S f^{\prime}\right)(x), \\
& \int_{\mathbb{Z}_{p}} f(x+t+1) d t-\int_{\mathbb{Z}_{p}} f(x+t) d t=f^{\prime}(x), \\
& \int_{\mathbb{Z}_{p}} f(-t) d t=\int_{\mathbb{Z}_{p}} f(t+1) d t .
\end{aligned}
$$

Moreover, for $f \in C^{2}\left(\mathbb{Z}_{p} \rightarrow \mathbb{C}_{p}\right)$, we have

$$
\frac{d}{d x} \int_{\mathbb{Z}_{p}} f(x+t) d t=\int_{\mathbb{Z}_{p}} f^{\prime}(x+t) d t
$$

for $x \in \mathbb{Z}_{p}([9$, p. 268]).

The Bernoulli polynomials $B_{n}(x)$ are defined by the generating function

$$
\frac{t e^{x t}}{e^{t}-1}=\sum_{n=0}^{\infty} \frac{B_{n}(x)}{n !} t^{n}
$$

When $x=0$, the numbers $B_{n}(0)=B_{n}$ are the ordinary Bernoulli numbers. It is known that the Bernoulli polynomials are expressed by using a Volkenborn integral:

$$
\int_{\mathbb{Z}_{p}}(x+t)^{n} d t=B_{n}(x) \quad(n \geq 0) .
$$

In particular, we have

$$
\int_{\mathbb{Z}_{p}} t^{n} d t=B_{n} \quad(n \geq 0)
$$

(e.g. [9, p. 271]).

Let $r$ be a positive integer and $x \in \mathbb{C}_{p}$. As a generalization of (2.6), we define multiple Bernoulli polynomials as

$$
S_{r}(x)=\frac{1}{r !} \int_{\mathbb{Z}_{p}^{r}}\left(x+t_{1}+\cdots+t_{r}\right)^{r} d t_{1} \ldots d t_{r},
$$

where $\int_{\mathbb{Z}_{p}^{r}}$ means $\underbrace{\int_{\mathbb{Z}_{p}} \cdots \int_{\mathbb{Z}_{p}}}_{r}$. From the multinomial expansion and equations 
(2.6) and (2.7), we have

$$
S_{r}(x)=\sum \frac{B_{k_{1}}(x) B_{k_{2}} \cdots B_{k_{r}}}{k_{1} ! \cdots k_{r} !},
$$

where the summation is over all non-negative integers $k_{1}, \ldots, k_{r}$ with $k_{1}+$ $\cdots+k_{r}=r$. Thus $S_{r}(x)$ is a polynomial with rational coefficients of degree $r$. We note that $S_{r}(x)$ is a special case of Barnes's multiple Bernoulli polynomials (cf. Ota [8, Section 2]).

3. Multiple $p$-adic log-gamma functions. For an integer $r \geq 0$, Endo [5] introduced the function $\varphi_{r}: \mathbb{C}_{p}^{\times} \rightarrow \mathbb{C}_{p}$ defined by

$$
\varphi_{r}(x)= \begin{cases}\frac{x^{r}}{r !}\left(\log _{p} x-\sum_{i=1}^{r} \frac{1}{i}\right) & (r \geq 1), \\ \log _{p} x & (r=0) .\end{cases}
$$

Using this function, he defined multiple $p$-adic log-gamma functions on $\mathbb{Z}_{p}$, which are generalizations of the logarithm of Morita's p-adic gamma function. Endo's multiple $p$-adic log-gamma functions will be dealt with in the last section.

From the definition, it is easily proved that for $r \geq 1$,

$$
x \varphi_{r-1}(x)=r \varphi_{r}(x)+\frac{x^{r}}{r !} .
$$

Since $\left(\log _{p} x\right)^{\prime}=1 / x$ for $x \in \mathbb{C}_{p}^{\times}$, we have $\frac{d}{d x} \varphi_{r}(x)=\varphi_{r-1}(x)$ for $r \geq 1$. Moreover, since $\log _{p}(x y)=\log _{p} x+\log _{p} y$ for all $x, y \in \mathbb{C}_{p}^{\times}$, we deduce that, for integers $r \geq 0$ and $k \geq 1$,

$$
\varphi_{r}(k x)=k^{r} \varphi_{r}(x)+\frac{(k x)^{r}}{r !} \log _{p} k .
$$

In particular, since $\log _{p} p=\log _{p}(-1)=0$, we have $\varphi_{r}(p x)=p^{r} \varphi_{r}(x)$ and $\varphi_{r}(-x)=(-1)^{r} \varphi_{r}(x)$.

LEMma 3.1. Let $f \in C^{r}\left(\mathbb{Z}_{p} \rightarrow \mathbb{C}_{p}\right)(r \geq 2)$. Then $F(x)=\int_{\mathbb{Z}_{p}} f(x+t) d t$ $\in C^{r-1}\left(\mathbb{Z}_{p} \rightarrow \mathbb{C}_{p}\right)$. Therefore, the integral

$$
\int_{\mathbb{Z}_{p}^{r}} f\left(t_{1}+\cdots+t_{r}\right) d t_{1} \ldots d t_{r}
$$

can be defined if $f \in C^{r}\left(\mathbb{Z}_{p} \rightarrow \mathbb{C}_{p}\right)$.

Proof. If $f \in C^{r}\left(\mathbb{Z}_{p} \rightarrow \mathbb{C}_{p}\right)$, then $S f \in C^{r}\left(\mathbb{Z}_{p} \rightarrow \mathbb{C}_{p}\right)$ (e.g. [10, Corollary 54.3]). Moreover, if $S f \in C^{r}\left(\mathbb{Z}_{p} \rightarrow \mathbb{C}_{p}\right)$, then $(S f)^{\prime} \in C^{r-1}\left(\mathbb{Z}_{p} \rightarrow \mathbb{C}_{p}\right)$ (cf. [10, Theorem 78.2]). By (2.1), we obtain the first part of the lemma. The second part can be proved by induction on $r$. 
Definition 3.2. For any integer $r \geq 0$ and $x \in \mathbb{C}_{p} \backslash \mathbb{Z}_{p}$, we define multiple p-adic log-gamma functions by

$$
\log \Gamma_{\mathrm{D}, r}(x)= \begin{cases}\int_{\mathbb{Z}_{p}^{r}} \varphi_{r}\left(x+t_{1}+\cdots+t_{r}\right) d t_{1} \ldots d t_{r} & (r \geq 1), \\ \log _{p} x & (r=0) .\end{cases}
$$

Since locally analytic functions are $C^{\infty}$-functions (e.g. [10, Corollary 29.11]), we have $\varphi_{r} \in C^{\infty}\left(\mathbb{Z}_{p} \rightarrow \mathbb{C}_{p}\right)$ for all $r \geq 0$. Therefore, by Lemma 3.1. this definition makes sense and $t \mapsto \log \Gamma_{\mathrm{D}, r}(x+t)$ is also a $C^{\infty}$-function from $\mathbb{Z}_{p}$ to $\mathbb{C}_{p}$ for a fixed $x \in \mathbb{C}_{p} \backslash \mathbb{Z}_{p}$. When $r=1$, we have

$$
\log \Gamma_{\mathrm{D}, 1}(x)=\int_{\mathbb{Z}_{p}}\left((x+t) \log _{p}(x+t)-(x+t)\right) d t \quad\left(x \in \mathbb{C}_{p} \backslash \mathbb{Z}_{p}\right)
$$

and this function is nothing but Diamond's $p$-adic log-gamma function $\log \Gamma_{\mathrm{D}}(x)$ (it was originally denoted by $G_{p}(x)$, see [4]). Diamond proved that $\log \Gamma_{\mathrm{D}}(x+1)-\log \Gamma_{\mathrm{D}}(x)=\log _{p} x$ for all $x \in \mathbb{C}_{p} \backslash \mathbb{Z}_{p}$.

We prove the following lemma which is needed to give properties of multiple $p$-adic log-gamma functions. This identity (3.3) has appeared in [10, p. 170] without proof, and we give its proof here.

Lemma 3.3. Let $k$ be a positive integer. For $f \in C^{1}\left(\mathbb{Z}_{p} \rightarrow \mathbb{C}_{p}\right)$, we have

$$
\int_{\mathbb{Z}_{p}} f(t) d t=\frac{1}{k} \sum_{i=0}^{k-1} \int_{\mathbb{Z}_{p}} f(i+k s) d s .
$$

Proof. From the definition of Volkenborn integrals, we have

$$
\begin{aligned}
\sum_{i=0}^{k-1} \int_{\mathbb{Z}_{p}} f(i+k s) d s & =\sum_{i=0}^{k-1} \lim _{N \rightarrow \infty} \frac{1}{p^{N}} \sum_{j=0}^{p^{N}-1} f(i+k j)=\lim _{N \rightarrow \infty} \frac{1}{p^{N}} \sum_{l=0}^{k p^{N}-1} f(l) \\
& =\sum_{i=0}^{k-1} \lim _{N \rightarrow \infty} \frac{1}{p^{N}} \sum_{j=0}^{p^{N}-1} f\left(i p^{N}+j\right) .
\end{aligned}
$$

Hence, by using the uniform convergence of the series, we obtain

$$
\begin{aligned}
\lim _{N \rightarrow \infty} \frac{1}{p^{N}} \sum_{j=0}^{p^{N}-1} f\left(i p^{N}+j\right) & =\lim _{N \rightarrow \infty} \frac{1}{p^{N}} \sum_{j=0}^{p^{N}-1} \lim _{M \rightarrow \infty} f\left(i p^{M}+j\right) \\
& =\lim _{N \rightarrow \infty} \frac{1}{p^{N}} \sum_{j=0}^{p^{N}-1} f(j)=\int_{\mathbb{Z}_{p}} f(t) d t
\end{aligned}
$$

for any integer $i$. This proves the lemma.

Now we give some properties of multiple $p$-adic log-gamma functions, which are generalizations of those of Diamond's $p$-adic log-gamma function (see [10, Theorem 60.2]). 
Proposition 3.4. Let $r$ and $k$ be positive integers. For $x \in \mathbb{C}_{p} \backslash \mathbb{Z}_{p}$, the following identities hold:

(i) $\log \Gamma_{\mathrm{D}, r}(x+1)-\log \Gamma_{\mathrm{D}, r}(x)=\log \Gamma_{\mathrm{D}, r-1}(x)$.

(ii) $\log \Gamma_{\mathrm{D}, r}(-x)=(-1)^{r} \log \Gamma_{\mathrm{D}, r}(x+r)$.

(iii) $\log \Gamma_{\mathrm{D}, r}(x)$

$$
=\sum_{i_{1}=0}^{k-1} \ldots \sum_{i_{r}=0}^{k-1} \log \Gamma_{\mathrm{D}, r}\left(\frac{x+i_{1}+\cdots+i_{r}}{k}\right)+\left(\log _{p} k\right) S_{r}(x) .
$$

In particular, when $k=p$, we have

$$
\log \Gamma_{\mathrm{D}, r}(x)=\sum_{i_{1}=0}^{p-1} \cdots \sum_{i_{r}=0}^{p-1} \log \Gamma_{\mathrm{D}, r}\left(\frac{x+i_{1}+\cdots+i_{r}}{p}\right) .
$$

Proof. Assertion (i) is easily proved by (2.3) and 2.5). By the identity $\varphi_{r}(-x)=(-1)^{r} \varphi_{r}(x)$ and $(2.4)$, we have

$$
\begin{aligned}
\log \Gamma_{\mathrm{D}, r}(-x) & =\int_{\mathbb{Z}_{p}^{r}} \varphi_{r}\left(-x+t_{1}+\cdots+t_{r}\right) d t_{1} \ldots d t_{r} \\
& =(-1)^{r} \int_{\mathbb{Z}_{p}^{r}} \varphi_{r}\left(x-t_{1}-\cdots-t_{r}\right) d t_{1} \ldots d t_{r} \\
& =(-1)^{r} \int_{\mathbb{Z}_{p}^{r}} \varphi_{r}\left(x+\left(t_{1}+1\right)+\cdots+\left(t_{r}+1\right)\right) d t_{1} \ldots d t_{r} \\
& =(-1)^{r} \log \Gamma_{\mathrm{D}, r}(x+r)
\end{aligned}
$$

and this proves (ii). Assertion (iii) follows by $(3.2)$ and Lemma 3.3 . In fact, $\log \Gamma_{\mathrm{D}, r}(x)=\int_{\mathbb{Z}_{p}^{r}} \varphi_{r}\left(x+t_{1}+\cdots+t_{r}\right) d t_{1} \ldots d t_{r}$

$$
\begin{aligned}
= & \frac{1}{k^{r}} \sum_{i_{1}=0}^{k-1} \ldots \sum_{i_{r}=0}^{k-1} \int_{\mathbb{Z}_{p}^{r}} \varphi_{r}\left(x+\left(i_{1}+k s_{1}\right)+\cdots+\left(i_{r}+k s_{r}\right)\right) d s_{1} \ldots d s_{r} \\
= & \frac{1}{k^{r}} \sum_{i_{1}=0}^{k-1} \ldots \sum_{i_{r}=0}^{k-1} \int_{\mathbb{Z}_{p}^{r}}\left(k^{r} \varphi_{r}\left(\frac{x+i_{1}+\cdots+i_{r}}{k}+s_{1}+\cdots+s_{r}\right)\right. \\
& \left.+\frac{\log _{p} k}{r !}\left(x+i_{1}+\cdots+i_{r}+k s_{1}+\cdots+k s_{r}\right)^{r}\right) d s_{1} \ldots d s_{r} \\
= & \sum_{i_{1}=0}^{k-1} \ldots \sum_{i_{r}=0}^{k-1} \log \Gamma_{\mathrm{D}, r}\left(\frac{x+i_{1}+\cdots+i_{r}}{k}\right) \\
& +\frac{1}{k^{r}} \sum_{i_{1}=0}^{k-1} \ldots \sum_{i_{r}=0}^{k-1} \frac{\log _{p} k}{r !} \int_{\mathbb{Z}_{p}^{r}}\left(x+i_{1}+\cdots+i_{r}+k s_{1}+\cdots+k s_{r}\right)^{r} d s_{1} \ldots d s_{r} .
\end{aligned}
$$


By using Lemma 3.3 again, we have

$$
\begin{aligned}
\log \Gamma_{\mathrm{D}, r}(x)= & \sum_{i_{1}=0}^{k-1} \ldots \sum_{i_{r}=0}^{k-1} \log \Gamma_{\mathrm{D}, r}\left(\frac{x+i_{1}+\cdots+i_{r}}{k}\right) \\
& +\frac{\log _{p} k}{r !} \int_{\mathbb{Z}_{p}^{r}}\left(x+t_{1}+\cdots+t_{r}\right)^{r} d t_{1} \ldots d t_{r} \\
= & \sum_{i_{1}=0}^{k-1} \ldots \sum_{i_{r}=0}^{k-1} \log \Gamma_{\mathrm{D}, r}\left(\frac{x+i_{1}+\cdots+i_{r}}{k}\right)+\left(\log _{p} k\right) S_{r}(x) .
\end{aligned}
$$

The last formula immediately follows because $\log _{p} p=0$.

4. Proof of the Main Theorem. We first give lemmas to prove our Main Theorem.

Lemma 4.1. For $f \in C^{2}\left(\mathbb{Z}_{p} \rightarrow \mathbb{C}_{p}\right)$, we have

$$
\int_{\mathbb{Z}_{p}}(t+1) f^{\prime}(t) d t=\int_{\mathbb{Z}_{p}} f(t) d t-\int_{\mathbb{Z}_{p}} \int_{\mathbb{Z}_{p}} f(x+t) d x d t .
$$

Proof. It is known that

$$
\int_{\mathbb{Z}_{p}}(t+1) f(t) d t=-\int_{\mathbb{Z}_{p}} S f(t) d t
$$

(cf. [10, p. 170]). By (2.2), we have

$$
\int_{\mathbb{Z}_{p}}(t+1) f^{\prime}(t) d t=-\int_{\mathbb{Z}_{p}}\left(S f^{\prime}\right)(t) d t=\int_{\mathbb{Z}_{p}} f(t) d t-\int_{\mathbb{Z}_{p}} \int_{\mathbb{Z}_{p}} f(x+t) d x d t
$$

and this proves the lemma.

$$
\begin{aligned}
& \text { Lemma 4.2. Let } f \in C^{r}\left(\mathbb{Z}_{p} \rightarrow \mathbb{C}_{p}\right)(r \geq 1) \text {. Then } \\
& \int_{\mathbb{Z}_{p}^{r}}\left(t_{i}+1\right) f\left(t_{1}+\cdots+t_{r}\right) d t_{1} \ldots d t_{r}=\int_{\mathbb{Z}_{p}^{r}}\left(t_{j}+1\right) f\left(t_{1}+\cdots+t_{r}\right) d t_{1} \ldots d t_{r}
\end{aligned}
$$

for any $1 \leq i, j \leq r$.

Proof. We only have to prove the case $r=2$ :

$$
\int_{\mathbb{Z}_{p}^{2}}\left(t_{1}+1\right) f\left(t_{1}+t_{2}\right) d t_{1} d t_{2}=\int_{\mathbb{Z}_{p}^{2}}\left(t_{2}+1\right) f\left(t_{1}+t_{2}\right) d t_{1} d t_{2}
$$

for $f \in C^{2}\left(\mathbb{Z}_{p} \rightarrow \mathbb{C}_{p}\right)$. We put $F_{t_{2}}\left(t_{1}\right)=f\left(t_{1}+t_{2}\right)$. Then

$$
S F_{t_{2}}\left(t_{1}\right)=S f\left(t_{1}+t_{2}\right)-S f\left(t_{2}\right) .
$$


By (4.2), the left-hand side of (4.4) is equal to

$$
\begin{aligned}
\int_{\mathbb{Z}_{p}^{2}}\left(t_{1}+1\right) F_{t_{2}}\left(t_{1}\right) d t_{1} d t_{2} & =-\int_{\mathbb{Z}_{p}} \int_{\mathbb{Z}_{p}}\left(S F_{t_{2}}\right)\left(t_{1}\right) d t_{1} d t_{2} \\
& =-\int_{\mathbb{Z}_{p}} \int_{\mathbb{Z}_{p}}\left((S f)\left(t_{1}+t_{2}\right)-(S f)\left(t_{2}\right)\right) d t_{1} d t_{2} \\
& =-\int_{\mathbb{Z}_{p}^{2}}(S f)\left(t_{1}+t_{2}\right) d t_{1} d t_{2}+\int_{\mathbb{Z}_{p}}(S f)\left(t_{2}\right) d t_{2} .
\end{aligned}
$$

On the other hand, the right-hand side of (4.4) is equal to

$$
\begin{aligned}
\int_{\mathbb{Z}_{p}}\left(t_{2}+1\right) \int_{\mathbb{Z}_{p}} f\left(t_{1}+t_{2}\right) d t_{1} d t_{2} & =\int_{\mathbb{Z}_{p}}\left(t_{2}+1\right)(S f)^{\prime}\left(t_{2}\right) d t_{2} \\
& =-\int_{\mathbb{Z}_{p}^{2}}(S f)\left(t_{1}+t_{2}\right) d t_{1} d t_{2}+\int_{\mathbb{Z}_{p}}(S f)\left(t_{2}\right) d t_{2}
\end{aligned}
$$

because of Lemma 4.1. As a consequence, equation (4.4) holds.

We are now in a position to prove our Main Theorem.

Proof of the Main Theorem. First we prove the uniqueness (ii). This actually follows from a more general result in [3, Section 1], but we give a proof to make the paper self-contained. We assume that strictly differentiable functions $f(x)$ and $g(x)$ satisfy conditions (A) and (B). Set $h(x)=f(x)-g(x)$. By (B), we have $r \int_{\mathbb{Z}_{p}} h(x+t) d t=(x-r) h^{\prime}(x)$. By (A), we have $h(x+1)=h(x)$ for all $x \in \mathbb{Z}_{p}$. Therefore $\int_{\mathbb{Z}_{p}} h(x+t) d t=h(x)$. Moreover, $h^{\prime}(x)=0$ because

$$
\lim _{n \rightarrow \infty} \frac{h\left(x+p^{n}\right)-h(x)}{p^{n}}=0 .
$$

As a consequence, $h(x)=0$, and this proves (ii).

Now we prove (i). We calculate the following integral in two ways:

$$
\int_{\mathbb{Z}_{p}^{r}}\left(x+t_{1}+\cdots+t_{r}\right) \varphi_{r-1}\left(x+t_{1}+\cdots+t_{r}\right) d t_{1} \ldots d t_{r} .
$$

By equation (3.1), we obtain

$$
\begin{aligned}
\int_{\mathbb{Z}_{p}^{r}}(x+ & \left.t_{1}+\cdots+t_{r}\right) \varphi_{r-1}\left(x+t_{1}+\cdots+t_{r}\right) d t_{1} \ldots d t_{r} \\
& =\int_{\mathbb{Z}_{p}^{r}}\left(r \varphi_{r}\left(x+t_{1}+\cdots+t_{r}\right)+\frac{\left(x+t_{1}+\cdots+t_{r}\right)^{r}}{r !}\right) d t_{1} \ldots d t_{r} \\
& =r \log \Gamma_{\mathrm{D}, r}(x)+S_{r}(x) .
\end{aligned}
$$


On the other hand, by Lemma 4.2 ,

$$
\begin{aligned}
\int_{\mathbb{Z}_{p}^{r}}\left(x+t_{1}+\cdots+t_{r}\right) & \varphi_{r-1}\left(x+t_{1}+\cdots+t_{r}\right) d t_{1} \ldots d t_{r} \\
= & \sum_{i=1}^{r} \int_{\mathbb{Z}_{p}^{r}}\left(t_{i}+1\right) \varphi_{r-1}\left(x+t_{1}+\cdots+t_{r}\right) d t_{1} \ldots d t_{r} \\
& +(x-r) \int_{\mathbb{Z}_{p}^{r}} \varphi_{r-1}\left(x+t_{1}+\cdots+t_{r}\right) d t_{1} \ldots d t_{r} \\
= & r \int_{\mathbb{Z}_{p}^{r}}\left(t_{1}+1\right) \varphi_{r-1}\left(x+t_{1}+\cdots+t_{r}\right) d t_{1} \ldots d t_{r} \\
& +(x-r) \int_{\mathbb{Z}_{p}^{r}} \varphi_{r-1}\left(x+t_{1}+\cdots+t_{r}\right) d t_{1} \ldots d t_{r} .
\end{aligned}
$$

By Lemma 4.1 and the relation $\varphi_{r}^{\prime}(x)=\varphi_{r-1}(x)$, we have

$$
\begin{aligned}
\int_{\mathbb{Z}_{p}^{r}}\left(t_{1}+1\right) \varphi_{r-1}\left(x+t_{1}\right. & \left.+\cdots+t_{r}\right) d t_{1} \ldots d t_{r} \\
= & \int_{\mathbb{Z}_{p}^{r}} \varphi_{r}\left(x+t_{1}+\cdots+t_{r}\right) d t_{1} \ldots d t_{r} \\
& -\int_{\mathbb{Z}_{p}} \int_{\mathbb{Z}_{p}^{r}} \varphi_{r}\left(x+t_{1}+\cdots+t_{r}+t\right) d t_{1} \ldots d t_{r} d t \\
= & \log \Gamma_{\mathrm{D}, r}(x)-\int_{\mathbb{Z}_{p}} \log \Gamma_{\mathrm{D}, r}(x+t) d t .
\end{aligned}
$$

Moreover, by 2.5),

$$
\begin{aligned}
\int_{\mathbb{Z}_{p}^{r}} \varphi_{r-1}\left(x+t_{1}+\cdots+t_{r}\right) d t_{1} \ldots d t_{r} & =\frac{d}{d x} \int_{\mathbb{Z}_{p}^{r}} \varphi_{r}\left(x+t_{1}+\cdots+t_{r}\right) d t_{1} \ldots d t_{r} \\
& =\left(\log \Gamma_{\mathrm{D}, r}\right)^{\prime}(x) .
\end{aligned}
$$

Therefore

$$
\begin{aligned}
& \int_{\mathbb{Z}_{p}^{r}}\left(x+t_{1}+\cdots+t_{r}\right) \varphi_{r-1}\left(x+t_{1}+\cdots+t_{r}\right) d t_{1} \ldots d t_{r} \\
& \quad=r \log \Gamma_{\mathrm{D}, r}(x)-r \int_{\mathbb{Z}_{p}} \log \Gamma_{\mathrm{D}, r}(x+t) d t+(x-r)\left(\log \Gamma_{\mathrm{D}, r}\right)^{\prime}(x) .
\end{aligned}
$$

Combining (4.6) and 4.7, we obtain 1.4.

5. Multiple $p$-adic log-gamma functions on $\mathbb{Z}_{p}$. In this last section, we deal with multiple $p$-adic log-gamma functions defined on $\mathbb{Z}_{p}$. For 
a continuous function $f: \mathbb{Z}_{p} \rightarrow \mathbb{C}_{p}$, we use the notation

$$
f^{*}(x)= \begin{cases}f(x) & \left(\text { if } x \in \mathbb{Z}_{p}^{\times}\right), \\ 0 & \left(\text { if } x \in p \mathbb{Z}_{p}\right) .\end{cases}
$$

It is clear that $f^{*}(x)$ is also continuous on $\mathbb{Z}_{p}$ and $\frac{d}{d x}\left(f^{*}\right)=\left(\frac{d}{d x} f\right)^{*}$ if $f$ is differentiable (cf. [5]). For an integer $r \geq 0$ and $x \in \mathbb{Z}_{p}$, we define multiple $p$-adic log-gamma functions on $\mathbb{Z}_{p}$ as

$$
\log \Gamma_{\mathrm{M}, r}(x)= \begin{cases}\int_{\mathbb{Z}_{p}^{r}} \varphi_{r}^{*}\left(x+t_{1}+\cdots+t_{r}\right) d t_{1} \ldots d t_{r} & (r \geq 1), \\ \log _{p}^{*}(x) & (r=0) .\end{cases}
$$

The function $\log \Gamma_{\mathrm{M}, r}(x)$ satisfies the difference equation

$$
\log \Gamma_{\mathrm{M}, r}(x+1)-\log \Gamma_{\mathrm{M}, r}(x)=\log \Gamma_{\mathrm{M}, r-1}(x) \quad\left(x \in \mathbb{Z}_{p}\right)
$$

for all $r \geq 1$. When $r=1$, the function $\log \Gamma_{\mathrm{M}, 1}(x)$ is the logarithm of Morita's $p$-adic gamma function, i.e. $\log \Gamma_{\mathrm{M}, 1}(x)=\log _{p} \Gamma_{p}(x)$ (e.g. [3, p. 370]).

REMARK 1. Endo [5, p. 45] introduced multiple $p$-adic log-gamma functions $G_{r}(x)$ for $r \geq 1$ and $x \in \mathbb{Z}_{p}$ as

$$
\begin{aligned}
& G_{r}(x) \\
= & \int_{\mathbb{Z}_{p}^{r}}\left[\varphi_{r}^{*}\left(x+t_{1}+\cdots+t_{r}\right)-\sum_{k=0}^{r}\left(\begin{array}{c}
x \\
r-k
\end{array}\right) \varphi_{k}^{*}\left(t_{1}+\cdots+t_{k}\right)\right] d t_{1} \ldots d t_{r} .
\end{aligned}
$$

He showed that the function $G_{r}$ satisfies not only the difference equation $G_{r+1}(x+1)-G_{r+1}(x)=G_{r}(x)$ but the good initial condition $G_{r}(0)=0$ for all $r \geq 1$ ([5, Theorem 5]). Therefore the function $G_{r}$ can be considered as a modification of (5.1), but for the sake of simplicity, we consider (5.1) in this paper.

The following proposition gives a relation between $\log \Gamma_{\mathrm{M}, r}(x)$ and $\log \Gamma_{\mathrm{D}, r}(x)$. This is a generalization of the known formula (e.g. [10, Theorem $60.2])$ :

$$
\log \Gamma_{\mathrm{M}, 1}(x)=\sum_{\substack{i=0 \\ p \nmid(x+i)}}^{p-1} \log \Gamma_{\mathrm{D}, 1}\left(\frac{x+i}{p}\right) \quad\left(x \in \mathbb{Z}_{p}\right) .
$$

Proposition 5.1. For a positive integer $r$ and $x \in \mathbb{Z}_{p}$, we have

$$
\log \Gamma_{\mathrm{M}, r}(x)=\sum_{\substack{i_{1}=0 \\ p \nmid\left(x+i_{1}+\cdots+i_{r}\right)}}^{p-1} \ldots \sum_{i_{r}=0}^{p-1} \log \Gamma_{\mathrm{D}, r}\left(\frac{x+i_{1}+\cdots+i_{r}}{p}\right) .
$$


Proof. By (3.3), we obtain

$$
\begin{aligned}
\log \Gamma_{\mathrm{M}, r}(x) & =\int_{\mathbb{Z}_{p}^{r}} \varphi_{r}^{*}\left(x+t_{1}+\cdots+t_{r}\right) d t_{1} \ldots d t_{r} \\
& =\frac{1}{p^{r}} \sum_{i_{1}=0}^{p-1} \cdots \sum_{i_{r}=0}^{p-1} \int_{\mathbb{Z}_{p}^{r}} \varphi_{r}^{*}\left(x+\left(i_{1}+p s_{1}\right)+\cdots+\left(i_{r}+p s_{r}\right)\right) d s_{1} \ldots d s_{r} \\
= & \frac{1}{p^{r}} \sum_{\substack{i_{1}=0 \\
p \nmid\left(x+i_{1}+\cdots+i_{r}\right)}}^{p-1} \cdots \sum_{i_{r}=0}^{p-1} \int_{\mathbb{Z}_{p}^{r}} \varphi_{r}\left(x+i_{1}+\cdots+i_{r}+p s_{1}+\cdots+p s_{r}\right) d s_{1} \ldots d s_{r} .
\end{aligned}
$$

By the equation $\varphi_{r}(p x)=p^{r} \varphi_{r}(x)$, we have

$$
\begin{aligned}
\log & \Gamma_{\mathrm{M}, r}(x) \\
& =\sum_{\substack{i_{1}=0 \\
p \nmid\left(x+i_{1}+\cdots+i_{r}\right)}}^{p-1} \ldots \sum_{\substack{i_{r}=0 \\
\mathbb{Z}_{p}^{r}}}^{p-1} \varphi_{r}\left(\frac{x+i_{1}+\cdots+i_{r}}{p}+s_{1}+\cdots+s_{r}\right) d s_{1} \ldots d s_{r} \\
& =\sum_{\substack{i_{1}=0 \\
p \nmid\left(x+i_{1}+\cdots+i_{r}\right)}}^{p-1} \cdots \sum_{i_{r}=0}^{p-1} \log \Gamma_{\mathrm{D}, r}\left(\frac{x+i_{1}+\cdots+i_{r}}{p}\right) .
\end{aligned}
$$

In the last part of this paper, we show that the function $\log \Gamma_{\mathrm{M}, r}(x)$ satisfies the following integro-differential equation similar to (1.4).

Proposition 5.2. For a positive integer $r$ and $x \in \mathbb{Z}_{p}$, we have

$$
\begin{aligned}
r \int_{\mathbb{Z}_{p}} \log \Gamma_{\mathrm{M}, r}(x+t) d t & \\
\quad & =(x-r)\left(\log \Gamma_{\mathrm{M}, r}\right)^{\prime}(x)-S_{r}(x)+\sum^{\prime} S_{r}\left(\frac{x+i_{1}+\cdots+i_{r}}{p}\right),
\end{aligned}
$$

where the summation is over all integers $i_{1}, \ldots, i_{r}$ with $0 \leq i_{l} \leq p-1$ $(1 \leq l \leq r)$ and $p \mid\left(x+i_{1}+\cdots+i_{r}\right)$.

This proposition is a generalization of the formula in [3, Proposition 2.4]:

$$
\int_{\mathbb{Z}_{p}} \log \Gamma_{\mathrm{M}, 1}(x+t) d t=(x-1)\left(\log \Gamma_{\mathrm{M}, 1}\right)^{\prime}(x)-x+\left\lceil\frac{x}{p}\right\rceil \quad\left(x \in \mathbb{Z}_{p}\right),
$$

where $\lceil x / p\rceil\left(x \in \mathbb{Z}_{p}\right)$ is the $p$-adic limit of the usual integer ceiling function $\left\lceil x_{n} / p\right\rceil$ as $x_{n} \rightarrow x$ through $x_{n} \in \mathbb{Z}$. In fact, when $r=1$ in Proposition 5.2 , 
then $S_{r}(x)=B_{1}(x)=x-1 / 2$ and the last term of $(5.2)$ is equal to

$$
\sum_{\substack{0 \leq i \leq p-1 \\ p \mid(x+i)}} B_{1}\left(\frac{x+i}{p}\right)=B_{1}\left(\left\lceil\frac{x}{p}\right\rceil\right)=\left\lceil\frac{x}{p}\right\rceil-\frac{1}{2} .
$$

In a way similar to the proof of the Main Theorem, we have

$$
\begin{aligned}
r & \int_{\mathbb{Z}_{p}} \log \Gamma_{\mathrm{M}, r}(x+t) d t \\
\quad & =(x-r)\left(\log \Gamma_{\mathrm{M}, r}\right)^{\prime}(x)-\frac{1}{r !} \int_{\mathbb{Z}_{p}^{r}}\left(x+t_{1}+\cdots+t_{r}\right)^{r *} d t_{1} \ldots d t_{r} .
\end{aligned}
$$

Therefore Proposition 5.2 follows from the next lemma.

Lemma 5.3. For a positive integer $r$ and $x \in \mathbb{Z}_{p}$, we have

$$
\int_{\mathbb{Z}_{p}^{r}}\left(x+t_{1}+\cdots+t_{r}\right)^{r *} d t_{1} \ldots d t_{r}=r ! S_{r}(x)-r ! \sum^{\prime} S_{r}\left(\frac{x+i_{1}+\cdots+i_{r}}{p}\right),
$$

where the summation is the same as in Proposition 5.2 .

Proof. By (3.3), we have

$$
\begin{aligned}
\int_{\mathbb{Z}_{p}^{r}}(x+ & \left.t_{1}+\cdots+t_{r}\right)^{r *} d t_{1} \ldots d t_{r} \\
= & \int_{\mathbb{Z}_{p}^{r}} \frac{1}{p^{r}} \sum_{i_{1}=0}^{p-1} \ldots \sum_{i_{r}=0}^{p-1}\left(x+\left(i_{1}+p s_{1}\right)+\cdots+\left(i_{r}+p s_{r}\right)\right)^{r *} d s_{1} \ldots d s_{r} \\
= & \int_{\mathbb{Z}_{p}^{r}} \frac{1}{p^{r}} \sum_{i_{1}=0}^{p-1} \ldots \sum_{i_{r}=0}^{p-1}\left(x+i_{1}+\cdots+i_{r}+p s_{1}+\cdots+p s_{r}\right)^{r} d s_{1} \ldots d s_{r} \\
& -\int_{\mathbb{Z}_{p}^{r}} \frac{1}{p^{r}} \sum_{i_{1}=0}^{p-1} \cdots \sum_{i_{r}=0}^{p-1}\left(x+i_{1}+\cdots+i_{r}+p s_{1}+\cdots+p s_{r}\right)^{r} d s_{1} \ldots d s_{r} \\
= & \int_{\mathbb{Z}_{p}^{r}}\left(x+t_{1}+\cdots+t_{r}\right)^{r} d t_{1} \ldots d t_{r} \\
& -\sum_{\mathbb{Z}_{p}^{r}}^{\prime}\left(\frac{x+i_{1}+\cdots+i_{r}}{p}+s_{1}+\cdots+s_{r}\right)^{r} d s_{1} \ldots d s_{r} \\
= & r ! S_{r}(x)-r ! \sum^{\prime} S_{r}\left(\frac{x+i_{1}+\cdots+i_{r}}{p}\right) .
\end{aligned}
$$

Acknowledgements. The author wishes to thank the referee for many useful comments and for pointing out some references. 


\section{References}

[1] E. W. Barnes, On the theory of the multiple gamma functions, Trans. Cambridge Philos. Soc. 19 (1904), 374-425.

[2] P. Cassou-Noguès, Analogues p-adiques des fonctions $\Gamma$-multiples, Astérisque 61 (1979), 43-55.

[3] H. Cohen and E. Friedman, Raabe's formula for p-adic gamma and zeta functions, Ann. Inst. Fourier (Grenoble) 58 (2008), 363-376.

[4] J. Diamond, The p-adic log gamma function and p-adic Euler constants, Trans. Amer. Math. Soc. 233 (1977), 321-337.

[5] M. Endo, p-adic multiple gamma functions, Comment. Math. Univ. St. Pauli 43 (1994), 35-54.

[6] H. Imai, Values of p-adic L-functions at positive integers and p-adic log multiple gamma functions, Tohoku Math. J. 45 (1993), 505-510.

[7] T. Kashio, On a p-adic analogue of Shintani's formula, J. Math. Kyoto Univ. 45 (2005), 99-128.

[8] K. Ota, On Kummer-type congruences for derivatives of Barnes' multiple Bernoulli polynomials, J. Number Theory 92 (2002), 1-36.

[9] A. M. Robert, A Course in p-adic Analysis, Grad. Texts in Math. 198, Springer, 2000 .

[10] W. H. Schikhof, Ultrametric Calculus, Cambridge Univ. Press, 1984.

[11] T. Shintani, On a Kronecker limit formula for real quadratic fields, J. Fac. Sci. Univ. Tokyo Sect. IA 24 (1977), 167-199.

[12] M.-F. Vignéras, L'équation fonctionnelle de la fonction zêta de Selberg du groupe modulaire PSL (2, Z), Astérisque 61 (1979), 235-249.

Ken Kamano

Department of General Education

Salesian Polytechnic

4-6-8, Oyamagaoka, Machida-city

Tokyo 194-0215, Japan

E-mail: kamano@salesio-sp.ac.jp

Received on 4.4.2009

and in revised form on 27.1.2010 